\title{
Rivaroxaban for Thromboprophylaxis after Hospitalization for Medical Illness
}

\author{
Alex C. Spyropoulos, M.D., Walter Ageno, M.D., Gregory W. Albers, M.D., \\ C. Gregory Elliott, M.D., Jonathan L. Halperin, M.D., William R. Hiatt, M.D., \\ Gregory A. Maynard, M.D., P. Gabriel Steg, M.D., Jeffrey I. Weitz, M.D., \\ Eunyoung Suh, Ph.D., Theodore E. Spiro, M.D., Elliot S. Barnathan, M.D., \\ and Gary E. Raskob, Ph.D., for the MARINER Investigators*
}

A B STR ACT

The authors' affiliations are listed in the Appendix. Address reprint requests to Dr. Spyropoulos at Northwell Health at Lenox Hill Hospital, 130 E. 77th St., New York, NY 10075, or at aspyropoul@ northwell.edu.

*A complete list of investigators and committee members in the MARINER trial is provided in the Supplementary Appendix, available at NEJM.org.

This article was published on August 26, 2018, at NEJM.org.

N Engl J Med 2018;379:1118-27. DOI: 10.1056/NEJMoa1805090 Copyright (c) 2018 Massachusetts Medical Society.

\section{BACKGROUND}

Patients who are hospitalized for medical illness remain at risk for venous thromboembolism after discharge, but the role of extended thromboprophylaxis in the treatment of such patients is a subject of controversy.

\section{METHODS}

In this randomized, double-blind trial, medically ill patients who were at increased risk for venous thromboembolism on the basis of a modified International Medical Prevention Registry on Venous Thromboembolism (IMPROVE) score of 4 or higher (scores range from 0 to 10, with higher scores indicating a higher risk of venous thromboembolism) or a score of 2 or 3 plus a plasma D-dimer level of more than twice the upper limit of the normal range (defined according to local laboratory criteria) were assigned at hospital discharge to either once-daily rivaroxaban at a dose of $10 \mathrm{mg}$ (with the dose adjusted for renal insufficiency) or placebo for 45 days. The primary efficacy outcome was a composite of symptomatic venous thromboembolism or death due to venous thromboembolism. The principal safety outcome was major bleeding.

\section{RESULTS}

Of the 12,024 patients who underwent randomization, 12,019 were included in the intention-to-treat analysis. The primary efficacy outcome occurred in 50 of 6007 patients $(0.83 \%)$ who were given rivaroxaban and in 66 of 6012 patients $(1.10 \%)$ who were given placebo (hazard ratio, $0.76 ; 95 \%$ confidence interval [CI], 0.52 to 1.09; $\mathrm{P}=0.14)$. The prespecified secondary outcome of symptomatic nonfatal venous thromboembolism occurred in $0.18 \%$ of patients in the rivaroxaban group and $0.42 \%$ of patients in the placebo group (hazard ratio, $0.44 ; 95 \% \mathrm{CI}, 0.22$ to 0.89). Major bleeding occurred in 17 of 5982 patients $(0.28 \%)$ in the rivaroxaban group and in 9 of 5980 patients $(0.15 \%)$ in the placebo group (hazard ratio, 1.88; $95 \% \mathrm{CI}, 0.84$ to 4.23$)$.

\section{CONCLUSIONS}

Rivaroxaban, given to medical patients for 45 days after hospital discharge, was not associated with a significantly lower risk of symptomatic venous thromboembolism and death due to venous thromboembolism than placebo. The incidence of major bleeding was low. (Funded by Janssen Research and Development; MARINER ClinicalTrials.gov number, NCT02111564.) 
P ATIENTS WHO ARE HOSPITALIZED FOR acute medical illnesses, such as heart failure, respiratory insufficiency, stroke, and infectious or inflammatory diseases, are at increased risk for venous thromboembolism. ${ }^{1}$ Validated risk scores that include additional factors, such as a lack of mobility, advanced age, cancer, previous venous thromboembolism, and elevated D-dimer levels, aid in the identification of patients who are at risk for symptomatic venous thromboembolism. ${ }^{2-4}$

Anticoagulant prophylaxis reduces the risk of in-hospital venous thromboembolism by 50 to $60 \%$ but is rarely continued after discharge in accordance with current guidelines. ${ }^{5,6}$ The risk of symptomatic venous thromboembolism, including fatal pulmonary embolism, in this population persists for 6 weeks or more after hospital discharge. ${ }^{2,7}$ However, studies of extended thromboprophylaxis have shown either excess major bleeding or a benefit that is based mainly on reducing the risk of asymptomatic deep-vein thrombosis..$^{8-11}$ Therefore, we performed a randomized trial of rivaroxaban treatment initiated at discharge and given for 45 days to medically ill patients who were at risk for venous thromboembolism. In our trial, we focused only on symptomatic or fatal events. ${ }^{12}$

\section{METHODS}

\section{TRIAL DESIGN AND OVERSIGHT}

The Medically Ill Patient Assessment of Rivaroxaban versus Placebo in Reducing Post-Discharge Venous Thrombo-Embolism Risk (MARINER) trial was a randomized, double-blind, placebocontrolled, multinational clinical trial. The rationale and design of the trial have been reported previously. ${ }^{12}$ An executive committee in collaboration with the sponsor (Janssen Research and Development) was responsible for the trial design, protocol, and oversight and served as the writing committee. The institutional review board or ethics committee at each of the 671 participating centers approved the protocol.

Coordination of study committees and support for trial execution were provided by an academic research organization (CPC Clinical Research), Worldwide Clinical Trials, and the academic leadership of the Antithrombotic Trials Leadership and Steering (ATLAS) Group. The data were collected by a contract research organization (Parexel) that was paid by the sponsor. An inde- pendent data and safety monitoring committee periodically reviewed trial outcomes and adverse events. The sponsor performed the statistical analysis in collaboration with the executive committee. The members of this committee, which included the authors, wrote all drafts of the manuscript and vouch for the accuracy and completeness of the data and analyses and for the fidelity of the trial to the protocol, which is available with the full text of this article at NEJM.org.

\section{PATIENTS}

Patients were eligible for participation in the trial if they were 40 years of age or older and had been hospitalized for at least 3 and not more than 10 consecutive days with one of the following conditions: heart failure with a left ventricular ejection fraction of $45 \%$ or less, acute respiratory insufficiency or exacerbation of chronic obstructive pulmonary disease, acute ischemic stroke, or acute infectious or inflammatory disease, including rheumatic diseases. Eligible patients also had to have additional risk factors for venous thromboembolism, as indicated by a total modified International Medical Prevention Registry on Venous Thromboembolism (IMPROVE) risk score of 4 or higher (scores range from 0 to 10 , with higher scores indicating a higher risk of venous thromboembolism; minimal clinically important difference, 2) or a risk score of 2 or 3 plus a plasma D-dimer level of more than twice the upper limit of the normal range, with $\mathrm{D}$-dimer measured locally and the normal range defined according to local laboratory criteria. Eligible patients must also have received thromboprophylaxis with low-molecular-weight heparin or unfractionated heparin during the index hospitalization.

Patients were excluded if they had a condition that was being treated with anticoagulant or dual antiplatelet therapy or if they had active cancer, a history of recent bleeding (within 3 months) or a high risk of bleeding, or other contraindications to rivaroxaban. The full list of inclusion and exclusion criteria and the criteria for the modified IMPROVE risk score ${ }^{2}$ are provided in Table S1 in the Supplementary Appendix, available at NEJM.org. All the patients provided written informed consent.

\section{TRIAL REGIMEN AND FOLLOW-UP}

Patients were randomly assigned in a 1:1 ratio to receive either rivaroxaban or placebo. Randomization was performed on the day of discharge 
from the hospital or the next day, with the use of an interactive Web-based system, with stratification according to country and creatinine clearance ( $\geq 30$ and $<50 \mathrm{ml}$ per minute or $\geq 50 \mathrm{ml}$ per minute). Randomization was balanced in permuted blocks of four. Patients were counseled about and instructed to promptly report symptoms or signs associated with deep-vein thrombosis, pulmonary embolism, and bleeding.

The rivaroxaban regimen was $10 \mathrm{mg}$ once daily for patients with a creatinine clearance of at least $50 \mathrm{ml}$ per minute or $7.5 \mathrm{mg}$ once daily for patients with a creatinine clearance of at least 30 but less than $50 \mathrm{ml}$ per minute. The first dose of rivaroxaban or placebo was given as soon as possible after randomization and not later than the next day. The trial agent was taken with or without food for 45 days. This duration was chosen because previous studies had shown that approximately $75 \%$ of post-hospital discharge venous thromboembolic events occur by 45 days after discharge. ${ }^{2,7}$

All the patients were contacted at approximately 7 days (range, 5 to 12), 21 days (range, 18 to 28 ), and 45 days (range, 45 to 49 ) after randomization, regardless of whether they continued to take rivaroxaban or placebo. At each contact, a review for suspected outcome events and assessment of symptoms, consisting of scripted questions (see the Supplementary Appendix), was completed. Counseling about the symptoms and signs of deep-vein thrombosis, pulmonary embolism, and bleeding was repeated, and data on adverse events and concomitant medications were collected. All the patients were contacted for safety follow-up on approximately day 75 (range, 70 to 80 ).

\section{OUTCOME MEASURES}

The primary efficacy outcome was the composite of any symptomatic venous thromboembolism (i.e., deep-vein thrombosis in the legs or nonfatal pulmonary embolism) or death related to venous thromboembolism (i.e., death due to pulmonary embolism or death in which pulmonary embolism could not be ruled out as the cause). The prespecified secondary efficacy outcomes were the two components of the primary outcome symptomatic nonfatal venous thromboembolism and death related to venous thromboembolism - analyzed separately; the composite of nonfatal symptomatic venous thromboembolism or death from any cause; the composite of nonfatal symp- tomatic venous thromboembolism, myocardial infarction, nonhemorrhagic stroke, or cardiovascular death (death due to a known cardiovascular cause or death in which a cardiovascular cause, including pulmonary embolism, could not be ruled out); and death from any cause.

The principal safety outcome was major bleeding. Other safety outcomes were nonmajor clinically relevant bleeding, other bleeding, and adverse events.

Major bleeding was defined as overt bleeding associated with a decrease in the hemoglobin level of $2 \mathrm{~g}$ per deciliter or more, bleeding that led to transfusion of 2 or more units of packed red cells or whole blood, bleeding that occurred in a critical site (i.e., intracranial, intraspinal, intraocular, pericardial, intraarticular, intramuscular with compartment syndrome, or retroperitoneal), or fatal bleeding. ${ }^{13}$ Nonmajor clinically relevant bleeding was defined as overt bleeding that did not meet the criteria for major bleeding but was associated with medical intervention, unscheduled contact (visit or telephone call) with a physician, temporary cessation of the trial regimen, or pain or impairment of activities of daily life. ${ }^{12}$ Other bleeding was defined as any other overt bleeding that did not meet the criteria for major or nonmajor clinically relevant bleeding.

A clinical events committee, the members of which were unaware of the trial-group assignments, adjudicated all suspected episodes of venous thromboembolism, bleeding, myocardial infarction, and stroke and all deaths, with the use of prespecified criteria. Death was adjudicated as being caused by pulmonary embolism, bleeding, cardiovascular disease, or other causes. Pulmonary embolism was considered the cause of death if there was objective documentation that pulmonary embolism caused the death or if the death could not be attributed to another documented cause and pulmonary embolism could not be ruled out. The criteria for the adjudication of the outcomes are provided in Table S2 in the Supplementary Appendix.

\section{STATISTICAL ANALYSIS}

The trial hypothesis was that rivaroxaban would be superior to placebo for the prevention of the primary efficacy outcome. ${ }^{12}$ The sample size was event-driven, with a targeted total number of events of the primary efficacy outcome of 161, which was determined under an assumption of a $40 \%$ lower relative risk with rivaroxaban than 


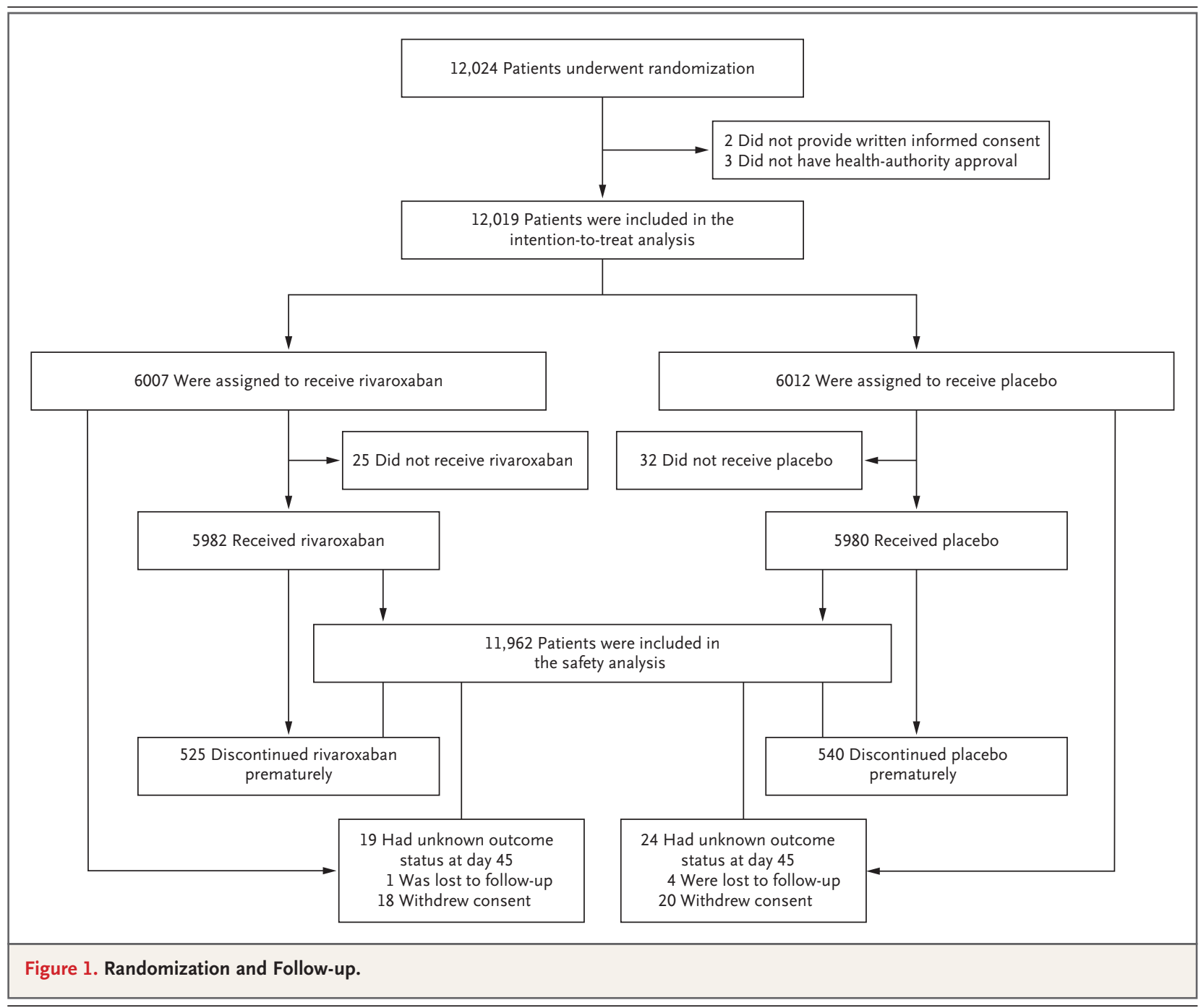

with placebo, a power of $90 \%$, and a two-sided significance level of 0.05 . We estimated that a total of approximately 8000 patients would be needed in order to observe 161 events, on the basis of an estimated incidence of $2.5 \%$ in the placebo group. We prespecified that randomization could be stopped at approximately 9000 patients for administrative reasons even if the targeted 161 events had not been observed. Because the blinded incidence of pooled events was lower than we had estimated, the protocol was amended to increase the maximum number of patients enrolled to 12,000. For patients with multiple events, only the first was counted toward the determination of the size of the trial population. One prespecified interim analysis was performed to evaluate futility. Thus, an adjustment of the final significance level was not done.
All the efficacy analyses were performed with the intention-to-treat population and included all data and outcomes from randomization through day 45 . The primary efficacy outcome was analyzed on the basis of the time from randomization to the first occurrence of symptomatic venous thromboembolism or venous thromboembolism-related death. The trial hypothesis was tested with a Cox proportional-hazards model, stratified according to creatinine clearance $(\geq 30$ and $<50 \mathrm{ml}$ per minute or $\geq 50 \mathrm{ml}$ per minute), with the randomly assigned regimen as the only covariate. Time-to-event curves were calculated with the Kaplan-Meier method.

If superiority of rivaroxaban for the prevention of the primary outcome was established, the secondary outcomes were to be tested sequentially with the use of the same Cox proportional- 


\begin{tabular}{|c|c|c|}
\hline Characteristic & $\begin{array}{l}\text { Rivaroxaban } \\
(\mathrm{N}=6007)\end{array}$ & $\begin{array}{l}\text { Placebo } \\
(\mathrm{N}=6012)\end{array}$ \\
\hline Mean age $-y r$ & 69.7 & 69.7 \\
\hline Age $\geq 75$ yr - no. (\%) & $2154(35.9)$ & $2140(35.6)$ \\
\hline Male sex — no. (\%) & $3130(52.1)$ & $3154(52.5)$ \\
\hline White race $-\% \dagger$ & $5782(96.3)$ & $5808(96.6)$ \\
\hline Mean weight — kg & 80.8 & 80.6 \\
\hline BMIț & 29.0 & 28.8 \\
\hline \multicolumn{3}{|l|}{ Creatinine clearance - no. (\%) } \\
\hline 30 to $<50 \mathrm{ml} / \mathrm{min}$ & $1098(18.3)$ & $1099(18.3)$ \\
\hline$\geq 50 \mathrm{ml} / \mathrm{min}$ & $4909(81.7)$ & $4913(81.7)$ \\
\hline \multicolumn{3}{|l|}{ Reason for index hospitalization — no./total no. (\%) } \\
\hline Heart failure & $2435 / 6003(40.6)$ & $2399 / 6011(39.9)$ \\
\hline Respiratory insufficiency or exacerbation of COPD & $1575 / 6003(26.2)$ & $1611 / 6011(26.8)$ \\
\hline Ischemic stroke & $860 / 6003(14.3)$ & $866 / 6011(14.4)$ \\
\hline Infectious disease & $1048 / 6003(17.5)$ & $1045 / 6011(17.4)$ \\
\hline Inflammatory disease & $85 / 6003(1.4)$ & $90 / 6011(1.5)$ \\
\hline Mean duration of index hospitalization - days & 6.7 & 6.7 \\
\hline Mean duration of in-hospital thromboprophylaxis — days & 6.2 & 6.2 \\
\hline History of VTE — no. (\%) & $765(12.7)$ & $748(12.4)$ \\
\hline History of cancer - no. (\%) & $488(8.1)$ & $533(8.9)$ \\
\hline ICU or CCU stay — no. (\%) & $3260(54.3)$ & $3240(53.9)$ \\
\hline Current lower-limb paralysis or paresis — no. (\%) & $1115(18.6)$ & $1122(18.7)$ \\
\hline \multicolumn{3}{|l|}{ Modified IMPROVE VTE risk score — no. (\%)』 } \\
\hline 2 & $2098(34.9)$ & $2151(35.8)$ \\
\hline 3 & $1886(31.4)$ & $1779(29.6)$ \\
\hline$\geq 4$ & $2019(33.6)$ & $2075(34.5)$ \\
\hline $\begin{array}{l}\text { D-Dimer level more than twice the upper limit of the normal } \\
\text { range during index hospitalization — no. (\%) } 9\end{array}$ & $4226(70.4)$ & $4239(70.5)$ \\
\hline Aspirin use - no. (\%) & $3159(52.6)$ & $3046(50.7)$ \\
\hline Thienopyridine use - no. (\%) & $360(6.0)$ & $388(6.5)$ \\
\hline \multicolumn{3}{|c|}{$\begin{array}{l}\text { * CCU denotes cardiac care unit, COPD chronic obstructive pulmonary disease, ICU intensive care unit, and VTE venous } \\
\text { thromboembolism. } \\
T \text { Race was reported by the patient. } \\
\text { The body-mass index (BMI) is the weight in kilograms divided by the square of the height in meters. } \\
\text { I Modified International Medical Prevention Registry on Venous Thromboembolism (IMPROVE) risk scores range from } \\
0 \text { to } 10 \text {, with higher scores indicating a higher risk of venous thromboembolism (minimal clinically important differ- } \\
\text { ence, 2). Eleven patients had protocol violations: three patients in the rivaroxaban group and seven patients in the } \\
\text { placebo group had a score of } 1 \text {, and one patient in the rivaroxaban group had a score of } 0 \text {. } \\
\text { The normal range for D-dimer level was defined according to the local laboratory criteria. }\end{array}$} \\
\hline
\end{tabular}

hazards model, in the following hierarchical order, each at an alpha level of 0.05 (two-sided): venous thromboembolism-related death; symptomatic venous thromboembolism; the composite of symptomatic venous thromboembolism or death from any cause; the composite of symptomatic venous thromboembolism, myocardial infarction, nonhemorrhagic stroke, or cardiovascular death; and death from any cause.

RESULTS

\section{PATIENTS AND TRIAL REGIMEN}

From June 2014 through January 2018, a total of 12,024 patients underwent randomization at 671 centers in 36 countries. The flow of the patients through the trial is shown in Figure 1. The baseline characteristics of the patients were similar in the two trial groups (Table 1). Permanent 


\begin{tabular}{|c|c|c|c|}
\hline Outcome & $\begin{array}{l}\text { Rivaroxaban } \\
\text { no. of pati }\end{array}$ & $\begin{array}{l}\text { Placebo } \\
\text { I no. (\%) }\end{array}$ & $\begin{array}{l}\text { Hazard Ratio } \\
(95 \% \mathrm{Cl}) \bar{\top}^{\circ}\end{array}$ \\
\hline \multicolumn{4}{|l|}{ Primary efficacy outcome } \\
\hline Symptomatic VTE or VTE-related death & $50 / 6007(0.83)$ & $66 / 6012(1.10)$ & $0.76(0.52-1.09) \doteqdot$ \\
\hline Creatinine clearance $\geq 50 \mathrm{ml} / \mathrm{min}, 10-\mathrm{mg}$ dose & $32 / 4909(0.65)$ & $48 / 4913(0.98)$ & $0.67(0.43-1.04)$ \\
\hline Creatinine clearance 30 to $<50 \mathrm{ml} / \mathrm{min}, 7.5-\mathrm{mg}$ dose & $18 / 1098(1.64)$ & $18 / 1099(1.64)$ & $1.00(0.52-1.92)$ \\
\hline \multicolumn{4}{|l|}{ Secondary efficacy outcomes } \\
\hline VTE-related death & $43 / 6007(0.72)$ & $46 / 6012(0.77)$ & $0.93(0.62-1.42)$ \\
\hline Symptomatic VTE & $11 / 6007(0.18)$ & $25 / 6012(0.42)$ & $0.44(0.22-0.89)$ \\
\hline Symptomatic VTE or death from any cause & $78 / 6007(1.30)$ & $107 / 6012(1.78)$ & $0.73(0.54-0.97)$ \\
\hline $\begin{array}{l}\text { Symptomatic VTE, myocardial infarction, nonhemorrhagic } \\
\text { stroke, or cardiovascular death }\end{array}$ & $94 / 6007(1.56)$ & $120 / 6012(2.00)$ & $0.78(0.60-1.02)$ \\
\hline Death from any cause & $71 / 6007(1.18)$ & $89 / 6012(1.48)$ & $0.80(0.58-1.09)$ \\
\hline \multicolumn{4}{|l|}{ Safety outcomes } \\
\hline Principal safety outcome: major bleeding & $17 / 5982(0.28)$ & $9 / 5980(0.15)$ & $1.88(0.84-4.23)$ \\
\hline Creatinine clearance $\geq 50 \mathrm{ml} / \mathrm{min}, 10-\mathrm{mg}$ dose & $13 / 4890(0.27)$ & $9 / 4890(0.18)$ & $1.44(0.62-3.37)$ \\
\hline Creatinine clearance 30 to $<50 \mathrm{ml} / \mathrm{min}, 7.5-\mathrm{mg}$ dose & $4 / 1092(0.37)$ & $0 / 1090$ & - \\
\hline \multicolumn{4}{|l|}{ Criteria for major bleeding $\int$} \\
\hline Hemoglobin decrease $\geq 2 \mathrm{~g} / \mathrm{dl}$ & $14 / 5982(0.23)$ & $6 / 5980(0.10)$ & $2.33(0.89-6.05)$ \\
\hline Transfusion of $\geq 2$ units of packed red cells & $11 / 5982(0.18)$ & $3 / 5980(0.05)$ & $3.66(1.02-13.10)$ \\
\hline Critical site & $3 / 5982(0.05)$ & $2 / 5980(0.03)$ & $1.50(0.25-8.97)$ \\
\hline Fatal & $2 / 5982(0.03)$ & $0 / 5980$ & - \\
\hline Nonmajor clinically relevant bleeding & $85 / 5982(1.42)$ & $51 / 5980(0.85)$ & $1.66(1.17-2.35)$ \\
\hline Other bleeding & $54 / 5982(0.90)$ & $34 / 5980(0.57)$ & $1.59(1.03-2.44)$ \\
\hline
\end{tabular}

* Symptomatic VTE included deep-vein thrombosis in the legs and nonfatal pulmonary embolism. VTE-related death included death due to pulmonary embolism and death in which pulmonary embolism could not be ruled out as the cause. Cardiovascular death included death due to a known cardiovascular cause and death in which a cardiovascular cause, including pulmonary embolism, could not be ruled out. $\dagger$ The confidence intervals have not been adjusted, and inferences drawn from the intervals may not be reproducible.

$\mathrm{P}=0.14$.

$\int$ Some patients may have had more than one criterion.

discontinuation of rivaroxaban or placebo before 45 days occurred in $8.9 \%$ of patients (Fig. 1). Temporary interruption of the regimen occurred in 176 patients (2.9\%) assigned to rivaroxaban and in 183 patients (3.1\%) assigned to placebo. Further details of treatment interruption and adherence are provided in Table S3 in the Supplementary Appendix.

\section{EFFICACY OUTCOMES}

The primary efficacy outcome of symptomatic venous thromboembolism or death related to venous thromboembolism occurred in 50 ( $0.83 \%)$ of 6007 patients in the rivaroxaban group and in $66(1.10 \%)$ of 6012 patients in the placebo group (hazard ratio, 0.76; 95\% confidence interval [CI], 0.52 to $1.09 ; \mathrm{P}=0.14$ ) (Table 2). The difference in risk (rivaroxaban minus placebo) was -0.27 percentage points ( $95 \% \mathrm{CI},-0.61$ to 0.08$)$. The time to the occurrence of the primary outcome and its two components are shown in Figure 2.

The incidence of the primary efficacy outcome both overall and according to creatinine clearance is shown in Table 2. Subgroup analyses for the primary efficacy outcome are shown in Figure S1 in the Supplementary Appendix. There were no significant interactions between subgroups and trial regimen.

Since superiority was not established in the primary efficacy analysis, the prespecified sec- 


\section{A Symptomatic VTE or VTE-Related Death}

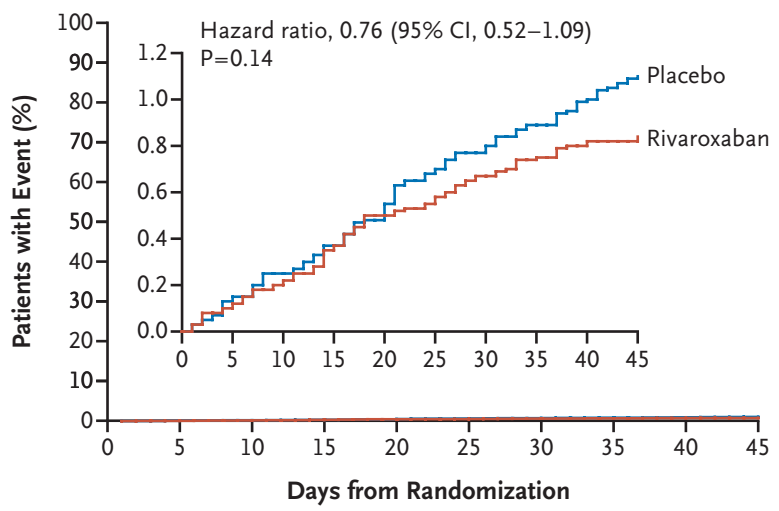

No. at Risk

Placebo

Rivaroxaban

$\begin{array}{llllllllll}6012 & 5989 & 5970 & 5959 & 5943 & 5922 & 5910 & 5902 & 5890 & 0\end{array}$ $\begin{array}{llllllllll}6007 & 5989 & 5972 & 5962 & 5948 & 5934 & 5927 & 5919 & 5913 & 0\end{array}$

\section{B VTE-Related Death}

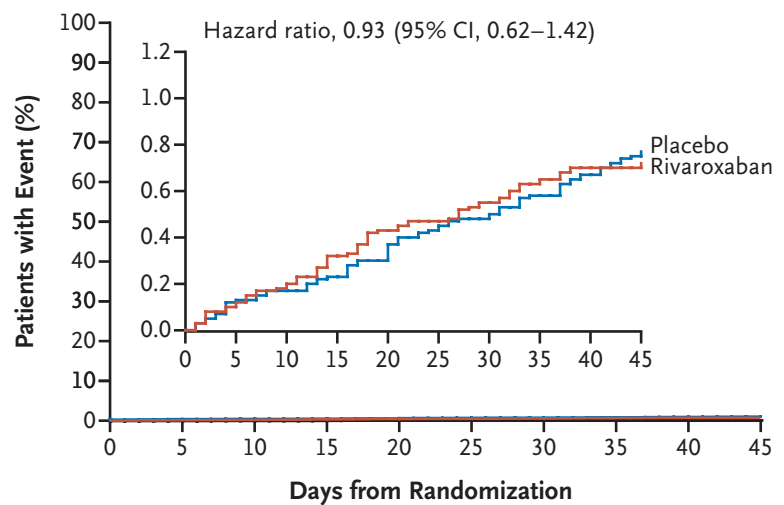

No. at Risk Placebo Rivaroxaban

$\begin{array}{llllllllll}6012 & 5993 & 5984 & 5976 & 5961 & 5949 & 5942 & 5934 & 5923 & 0\end{array}$ $\begin{array}{llllllllll}6007 & 5991 & 5980 & 5971 & 5957 & 5950 & 5943 & 5930 & 5925 & 0\end{array}$

\section{Symptomatic VTE}

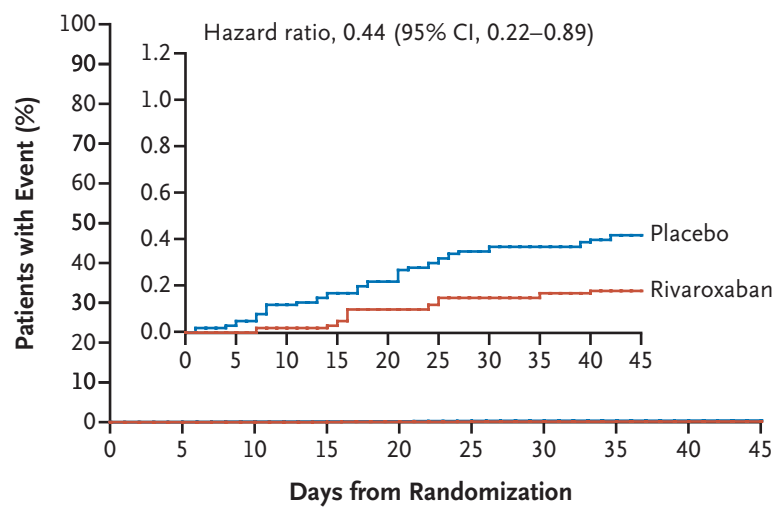

No. at Risk

Placebo

Rivaroxaban $\begin{array}{llllllllll}6012 & 5988 & 5962 & 5952 & 5939 & 5909 & 5898 & 5895 & 5886 & 0\end{array}$ $\begin{array}{llllllllll}6007 & 5989 & 5966 & 5960 & 5947 & 5927 & 5921 & 5916 & 5913 & 0\end{array}$
Figure 2. Kaplan-Meier Cumulative Event Rates for the Primary Efficacy Outcome and Its Components. Shown are cumulative event rates for the composite outcome of symptomatic venous thromboembolism (VTE) (deep-vein thrombosis in the legs or nonfatal pulmonary embolism) or VTE-related death (death due to pulmonary embolism or death in which pulmonary embolism could not be ruled out as the cause) (Panel A), VTE-related death (Panel B), or symptomatic VTE (Panel C). In each panel, the inset shows the same data on an expanded $y$ axis.

ondary efficacy outcomes were assessed as exploratory analyses without adjustment for multiplicity (Table 2). The time to the occurrence of each secondary outcome is shown in Figures 2 and 3.

\section{SAFETY OUTCOMES}

The principal safety outcome of major bleeding occurred in $17(0.28 \%)$ of 5982 patients in the rivaroxaban group and in $9(0.15 \%)$ of 5980 patients in the placebo group (hazard ratio, 1.88; 95\% CI, 0.84 to 4.23) (Table 2). The difference in risk (rivaroxaban minus placebo) was 0.13 percentage points ( $95 \%$ CI, -0.03 to 0.30 ). The incidence of major bleeding according to the prespecified stratification based on creatinine clearance is shown in Table 2.

Subgroup analyses of major bleeding are shown in Figure S2 in the Supplementary Appendix. There were no significant interactions between the trial regimen and any subgroup variable, with the exception of the duration of the index hospitalization $(\mathrm{P}=0.02)$ and in-hospital receipt of thromboprophylaxis $(\mathrm{P}=0.03)$.

The incidence of clinically relevant nonmajor bleeding and other bleeding is shown in Table 2 . Adverse events occurred with similar frequency in the rivaroxaban group and the placebo group (Tables S4, S5, and S6 in the Supplementary Appendix). A total of 160 patients died during the 45 -day treatment phase (71 in the rivaroxaban group and 89 in the placebo group). The causes of death are given in Table S7 in the Supplementary Appendix.

\section{DISCUSSION}

In this trial involving medically ill patients, rivaroxaban treatment that was started at the time of discharge from the hospital and continued for 
Figure 3. Kaplan-Meier Cumulative Event Rates for Composite Secondary Outcomes and Death from Any Cause.

Shown are cumulative event rates for the composite of symptomatic VTE or death from any cause (Panel A), the composite of symptomatic VTE, myocardial infarction, nonhemorrhagic stroke, or cardiovascular death (death due to a known cardiovascular cause or death in which a cardiovascular cause, including pulmonary embolism, could not be ruled out) (Panel B), and death from any cause (Panel C). In each panel, the inset shows the same data on an expanded $y$ axis.

45 days was not associated with a significantly lower risk of the primary efficacy outcome of fatal or symptomatic venous thromboembolism than placebo. Therefore, all the subsequent efficacy analyses were exploratory. Although rivaroxaban had no effect on the risk of venous thromboembolism-related death, it was associated with fewer symptomatic venous thromboembolic events than placebo (risk difference, -0.24 percentage points). The incidence of major bleeding during treatment with rivaroxaban was low $(0.28 \%$; difference in risk vs. placebo, 0.13 percentage points). These differences in risk suggest that the number of patients needed to treat to prevent one symptomatic venous thromboembolism event is 430 , whereas the number needed to cause one major bleed is 856 . Thus, although the benefit-risk decision for the individual patient is finely tuned, the implementation of extended thromboprophylaxis with appropriate selection of medically ill patients may reduce the health burden of nonfatal venous thromboembolism in this population.

The rationale for extended thromboprophylaxis is to prevent symptomatic deep-vein thrombosis and nonfatal and fatal pulmonary embolism. Although we observed fewer symptomatic venous thromboembolic events with rivaroxaban than with placebo, no significant difference in venous thromboembolism-related mortality was observed. This observation is consistent with previously published trials of either short- or extended-duration prophylaxis in medical patients. ${ }^{8-11,14,15}$ The incidence of venous thromboembolism-related death in the placebo group $(0.77 \%)$ was higher than in trials of other direct oral anticoagulants. ${ }^{8,10}$ Most of these deaths were

\section{A Symptomatic VTE or Death from Any Cause}

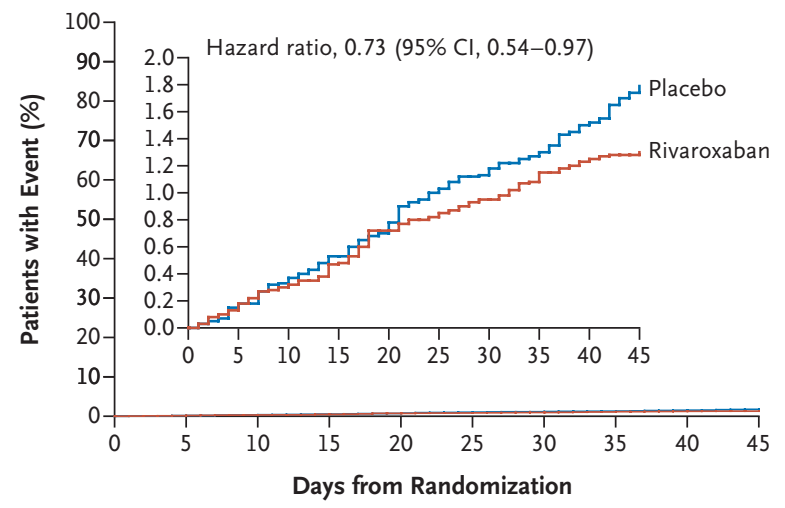

No. at Risk

Placebo

$\begin{array}{llllllllll}6012 & 5989 & 5974 & 5963 & 5945 & 5929 & 5918 & 5910 & 5897 & 0\end{array}$

Rivaroxaban

$\begin{array}{llllllllll}6007 & 5989 & 5976 & 5965 & 5950 & 5938 & 5931 & 5919 & 5913 & 0\end{array}$

\section{B Symptomatic VTE, Myocardial Infarction, Nonhemorrhagic Stroke,} or Cardiovascular Death

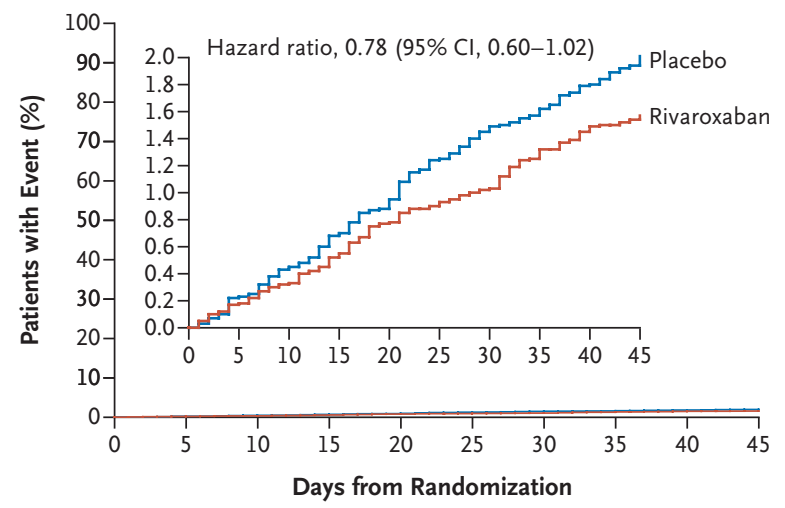

No. at Risk

Placebo

$\begin{array}{llllllllll}6012 & 5984 & 5964 & 5947 & 5928 & 5904 & 5886 & 5877 & 5862 & 0\end{array}$

Rivaroxaban

C Death from Any Cause

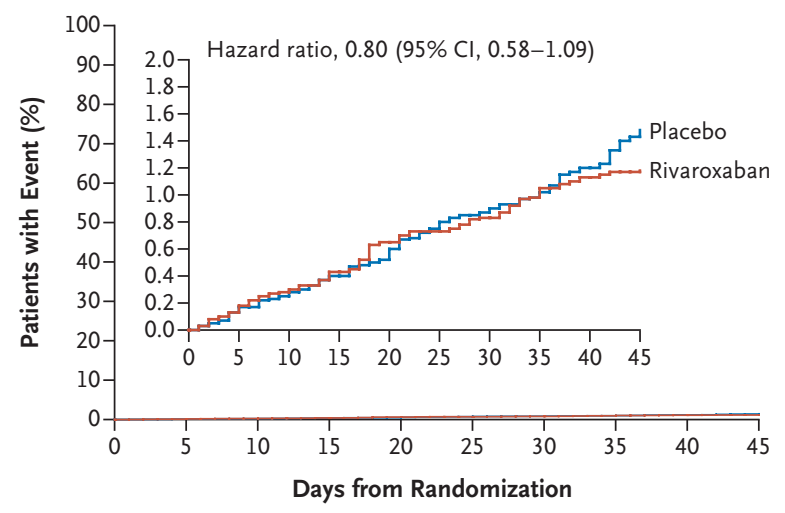

No. at Risk

Placebo

$\begin{array}{llllllllll}6012 & 5993 & 5984 & 5976 & 5961 & 5949 & 5942 & 5934 & 5923 & 0\end{array}$

Rivaroxaban 
sudden deaths in which pulmonary embolism could not be ruled out. The definition of venous thromboembolism-related death has varied across contemporary trials of extended thromboprophylaxis, and not all these trials have included unexplained sudden death..$^{8-10}$ The broader definition we used probably included some deaths that were not due to pulmonary embolism, which suggests that sudden death of unknown cause is not sufficiently specific for inclusion in the definition of fatal pulmonary embolism. Conversely, death from any cause includes death from thrombotic causes other than pulmonary embolism, which may explain why the composite outcome of symptomatic venous thromboembolism and death from any cause was less frequent in the rivaroxaban group.

In a previous trial involving medically ill patients, treatment with rivaroxaban at a dose of $10 \mathrm{mg}$ once daily started in the hospital and continued for 35 days reduced venous thromboembolism but increased major bleeding. ${ }^{9}$ Therefore, one goal of the current trial was to improve the safety of rivaroxaban in this population. This goal was achieved, as shown by lower incidences of major bleeding than in the previous trial. ${ }^{9}$ Safety was enhanced by initiating rivaroxaban at discharge, reducing the dose to $7.5 \mathrm{mg}$ daily in patients with moderate renal impairment, and excluding patients who were identified as being at high risk for bleeding in the previous trial. ${ }^{9}$ The latter group included patients who had active cancer or gastrointestinal ulcer, bronchiectasis, or bleeding in the previous 3 months or were receiving dual antiplatelet therapy.

Patients with renal insufficiency have a higher incidence of thrombotic and bleeding events ${ }^{16,17}$ than patients with normal renal function. In the previous trial, ${ }^{9}$ rivaroxaban at $10 \mathrm{mg}$ daily was effective in patients with moderate renal insufficiency but was associated with increased bleeding. In the current trial, the 7.5-mg daily dose of rivaroxaban given to patients with moderate renal insufficiency was associated with a low incidence of bleeding but not with a lower risk of the primary efficacy outcome than placebo. Similarly, the betrixaban dose-reduction strategy in medically ill patients showed less efficacy. ${ }^{10}$

This large trial of extended thromboprophylaxis in medically ill patients had strengths and limitations. The strengths included the use of symptomatic events as the primary efficacy out- come, a low rate of loss to follow-up, and independent event adjudication. Despite the use of a validated risk score and elevated D-dimer levels to enrich the rate of primary events, the incidence in the placebo group was $1.1 \%$ rather than the expected $2.5 \%$. Given the expected increase in D-dimer levels with age, it is possible that the cutoff we used for this assay (i.e., twice the upper limit of the normal range) led to the inclusion of participants whose risk was lower than expected. The low incidence prompted the decision to stop enrollment before accumulation of the prespecified 161 patients with primary events. Other limitations included the difficulty in defining venous thromboembolism-related death and the possible underdosing of patients with moderate renal impairment. In this trial, we did not record all the patients who were assessed for inclusion and from among whom the population that underwent randomization was selected. However, previous studies indicate that our inclusion criteria would represent approximately 25 to $30 \%$ of all hospitalized medical patients. ${ }^{3,4,18}$

In conclusion, our trial did not show a significant benefit of this rivaroxaban regimen started at hospital discharge with regard to the composite outcome of fatal or symptomatic venous thromboembolism in medically ill patients. Given the relatively low incidence of events despite the enrichment strategy and the lack of effect on venous thromboembolism-related death, the usefulness of extended thromboprophylaxis remains uncertain. Future studies should more accurately identify deaths caused by thrombotic mechanisms and focus on the patients who are at highest risk and who may benefit from anticoagulant prophylaxis.

\section{Supported by Janssen Research and Development.}

Dr. Spyropoulos reports receiving advisory board fees from Daiichi Sankyo and Portola, grant support, consulting fees, and advisory board fees from Boehringer Ingelheim and Janssen, consulting fees and advisory board fees from Bayer, and a stipend from ATLAS Group (Colorado Prevention Center); Dr. Ageno, receiving grant support and advisory board fees from Bayer and BMS Pfizer and advisory board fees from Portola, Daiichi Sankyo, Aspen, Boehringer Ingelheim, and Sanofi; Dr. Albers, receiving consulting fees from Bayer; Dr. Elliott, receiving fees for serving on a steering committee from Bayer and lecture fees from the University of Cincinnati and Spectrum Health; Dr. Halperin, receiving consulting fees from Boehringer Ingelheim, Daiichi Sankyo, Pfizer, ATLAS Group (Colorado Prevention Center), Johnson \& Johnson, and Ortho-McNeil-Janssen; Dr. Hiatt, receiving grant support from Janssen and Bayer; Dr. Steg, receiving grant support and fees for serving on a steering committee from Bayer/Janssen, grant support and lecture fees from Merck, grant support, consulting fees, lecture fees, and fees for serving as 
cochair of the ODYSSEY outcomes trial and the SCORED trial from Sanofi, grant support and fees for serving as chair of the CLARIFY registry from Servier, consulting fees and fees for serving on the executive steering committee for the REDUCE IT trial from Amarin, consulting fees and lecture fees from Amgen, consulting fees, lecture fees, and fees for critical event committee work from Bristol-Myers Squibb, fees for serving on the executive steering committee of the REDUAL PCI trial from Boehringer Ingelheim, fees for critical event committee work from Pfizer, consulting fees and fees for serving on the executive steering committee for the PARADISE MI trial from Novartis, consulting fees from Regeneron and Lilly, and consulting fees and fees for serving as cochair of the THEMIS trial from AstraZeneca; Dr. Weitz, receiving consulting fees and honoraria from Bayer, Boehringer Ingelheim, Bristol-Myers Squibb, Daiichi Sankyo, Ionis, Janssen, Merck, Novartis, Pfizer and Portola; Dr. Suh and Dr. Barnathan, being employed by Janssen Research and Development and owning stock in Johnson \& Johnson; Dr. Spiro, being employed by and owning shares in Bayer U.S.; and Dr. Raskob, receiving consulting fees from Bayer, BMS, Boehringer Ingelheim, Eli Lilly, Portola, and Novartis and consulting fees and honoraria from Daiichi Sankyo and Pfizer. No other potential conflict of interest relevant to this article was reported.

Disclosure forms provided by the authors are available with the full text of this article at NEJM.org.

A data sharing statement provided by the authors is available with the full text of this article at NEJM.org.

We thank Robert Achenbach, John Albanese, Peter DiBattiste, Wentao Lu, Chiara Sugarmann, and Daniel Sullivan, who supported the writing meeting of the executive committee, and the many patients and their families who participated in this trial.

Dr. Weitz holds a chair in thrombosis from the Canada Research Chairs Program and a chair in cardiovascular research from the Heart and Stroke Foundation of Canada.

\section{APPENDIX}

The authors' affiliations are as follows: the Donald and Barbara Zucker School of Medicine at Hofstra/Northwell, Feinstein Institute for Medical Research, and the Department of Medicine, Anticoagulation and Clinical Thrombosis Services, Northwell Health at Lenox Hill Hospital (A.C.S.), and the Cardiovascular Institute, Mount Sinai Medical Center (J.L.H.) — all in New York; the Department of Medicine and Surgery, University of Insubria, Varese, Italy (W.A.); the Stanford Stroke Center, Stanford University Medical Center, Stanford (G.W.A.), and the University of California at Davis, Sacramento (G.A.M.) - both in California; the Department of Medicine, Intermountain Medical Center, and Department of Medicine, University of Utah, Salt Lake City (C.G.E.); Division of Cardiology, University of Colorado School of Medicine, and CPC Clinical Research, Aurora (W.R.H.); Département Hospitalo-Universitaire FIRE (Fibrose Inflammation Remodelage), University Paris Diderot, Assistance Publique-Hôpitaux de Paris, and INSERM Unité 1148, Paris (P.G.S.); Imperial College, Royal Brompton Hospital, London (P.G.S.); McMaster University and the Thrombosis and Atherosclerosis Research Institute - both in Hamilton, ON, Canada (J.I.W.); Janssen Research and Development, Raritan (E.S., E.S.B.), and the Thrombosis and Hematology Therapeutic Area, Clinical Development, Pharmaceuticals, Bayer U.S., Whippany (T.E.S.) - both in New Jersey; and the College of Public Health, University of Oklahoma Health Sciences Center, Oklahoma City (G.E.R.).

\section{REFERENCES}

1. Cohen AT, Alikhan R, Arcelus JI, et al. Assessment of venous thromboembolism risk and the benefits of thromboprophylaxis in medical patients. Thromb Haemost 2005;94:750-9.

2. Spyropoulos AC, Anderson FA Jr, FitzGerald G, et al. Predictive and associative models to identify hospitalized medical patients at risk for VTE. Chest 2011;140:706-14.

3. Gibson CM, Spyropoulos AC, CohenAT, et al. The IMPROVEDD VTE risk score: incorporation of D-dimer into the IMPROVE score to improve venous thromboembolism risk stratification. TH Open2017;1(1):e56-e65.

4. Mahan CE, Liu Y, Turpie AG, et al. External validation of a risk assessment model for venous thromboembolism in the hospitalised acutely-ill medical patient (VTE-VALOURR). Thromb Haemost 2014;112:692-9.

5. Kahn SR, Lim W, Dunn AS, et al. Prevention of VTE in nonsurgical patients: Antithrombotic Therapy and Prevention of Thrombosis, 9th ed: American College of Chest Physicians Evidence-Based Clinical Practice Guidelines. Chest 2012; 141:Suppl 2:e195S-e226S.

6. Mahan CE, Fisher MD, Mills RM, et al. Thromboprophylaxis patterns, risk factors, and outcomes of care in the med- ically ill patient population. Thromb Res 2013;132:520-6.

7. Hull RD, Merali T, Mills A, Stevenson AL, Liang J. Venous thromboembolism in elderly high-risk medical patients: time course of events and influence of risk factors. Clin Appl Thromb Hemost 2013;19:357-62. 8. Goldhaber SZ, Leizorovicz A, Kakkar AK, et al. Apixaban versus enoxaparin for thromboprophylaxis in medically ill patients. N Engl J Med 2011;365:2167-77.

9. Cohen AT, Spiro TE, Büller HR, et al. Rivaroxaban for thromboprophylaxis in acutely ill medical patients. N Engl J Med 2013;368:513-23.

10. Cohen AT, Harrington RA, Goldhaber $\mathrm{SZ}$, et al. Extended thromboprophylaxis with betrixaban in acutely ill medical patients. N Engl J Med 2016;375:534-44.

11. Hull RD, Schellong SM, Tapson VF, et al. Extended-duration venous thromboembolism prophylaxis in acutely ill medical patients with recently reduced mobility: a randomized trial. Ann Intern Med 2010;153:8-18

12. Raskob GE, Spyropoulos AC, Zrubek J, et al. The MARINER trial of rivaroxaban after hospital discharge for medical patients at high risk of VTE: design, rationale, and clinical implications. Thromb Haemost 2016;115:1240-8.

13. Schulman S, Kearon C. Definition of major bleeding in clinical investigations of antihemostatic medicinal products in non-surgical patients. J Thromb Haemost 2005;3:692-4

14. Kakkar AK, Cimminiello C, Goldhaber SZ, Parakh R, Wang C, Bergmann J-F. Low-molecular-weight heparin and mortality in acutely ill medical patients. N Engl J Med 2011;365:2463-72.

15. Gärdlund B. Randomised, controlled trial of low-dose heparin for prevention of fatal pulmonary embolism in patients with infectious diseases. Lancet 1996; 347:1357-61.

16. Wattanakit K, Cushman M, StehmanBreen C, Heckbert SR, Folsom AR. Chronic kidney disease increases risk for venous thromboembolism. J Am Soc Nephrol 2008;19:135-40.

17. Lutz J, Menke J, Sollinger D, Schinzel $\mathrm{H}$, Thürmel K. Haemostasis in chronic kidney disease. Nephrol Dial Transplant 2014;29:29-40.

18. Rosenberg D, Eichorn A, Alarcon M, McCullagh L, McGinn T, Spyropoulos AC. External validation of the risk assessment model of the International Medical Prevention Registry on Venous Thromboembolism (IMPROVE) for medical patients in a tertiary health system. J Am Heart Assoc 2014;3(6):e001152.

Copyright (c) 2018 Massachusetts Medical Society. 